\title{
A Study On Database Structure Of Prosumer System For Online Analysis
}

\section{Hyun-Sung Lim* ,Gil-Mok Shong, Gun-Ho Yi ,Jae-Hyun Kim ,Sung-Koo Cho, Pil-Sung Woo}

Professor, Smart Electricity, Korea Polytechnic, Gimje, Jeollabuk-do, 54352, Korea

*Corresponding author. 1hs0218@kopo.ac.kr

Article History:Received:11 november 2020; Accepted: 27 December 2020; Published online: 05 April 2021

Abstract: Energy prosumer can not only save on electrical bills but also make profits by selling energy produced. To develop online prosumer platform, this paper present database structure of each prosumer system(Photovoltaic system, Energy Storage System, Wind power system, EV Charger system). The cause of abnormalities in prosumer facilities and designed the database and developed the reference model by big data measured from the hardware. The data was preprocessed all values from the acquired data between 0 and 1 and training set and test set generated for the data transposition process. For applied AI algorithms, this paper adapted DNN(Deep Neural Network) method. This paper used Precision, Recall, Accuracy, F1-score. The result of experiment is high value of each expression. Recall value is high values and this expression is important to safety area focus. This database structure will provide online prosumer platform system and batter prediction safety system.

Keywords: Prosumer, Database, AI algorithm, Deep Neural Network, Online platform.

\section{Introduction}

A prosumer is a customer who want to buy high-quality products or equipment. The word is formed from "producer" and "consumer". Energy prosumer can produce and sell electrical energy. This word used by futurist Alvin Toffler in the third wave, it is characterized by switching to a producer if energy production is greater than consumption and a consumer if energy consumption is greater than production. Energy prosumer can not only save on electrical bills but also make profits by selling energy produced [1 5]. This system require power transactions between individual producers. The government is working on system to revitalize prosumer while implementing the 3020 renewable energy policy. In Britain, the operation of Piclo, a web-based power trading platform, links and matches power producers and consumers every 30 minutes. Power generation companies and consumers present transaction prices and conditions, and the system in which transactions are made. Germany operated a trading platform between neighbors. It connected owners of solar power facilities by using platforms and shared supply power online. In order to operate the platform, it is important to transfer online data from the prosumer facilities [6 8]. This paper, we present data structure of prosumer system for online platform. This work is useful for studying online prosumer platform. Firstly, we analyze the causes of abnormalities in prosumer facilities and designed the database and developed the reference model by big data measured from the hardware. Finally, The data was preprocessed all values from the acquired data between 0 and 1 and the training set and test set generated for the data transposition process[11-13].

\section{Database design and development for data analysis}

In order to analyze the cause of abnormalities in the prosumer systems, it needs reference model from data with hardware. Measured data should be big data and designed database. Generally, prosumer system is ESS(energy storage system), Wind power system,. Photovoltaic system, EV charger system. Data acquired from each facilities contain Min-value, Max-value, Avg-value, RMS-value. This paper used MS-sql database and the table was as follows [Figure 1].

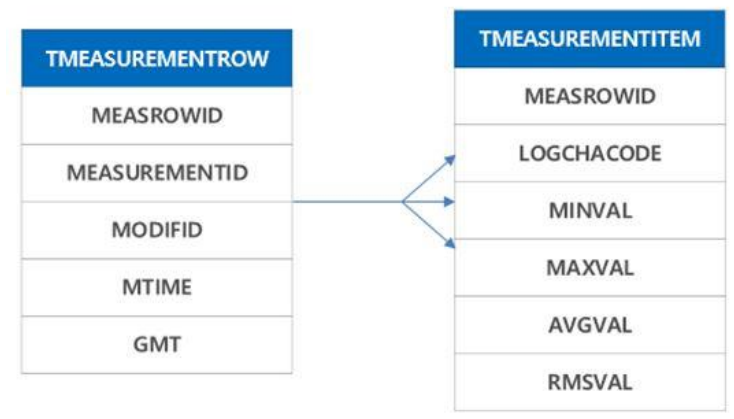

Figure 1 Database table structure

*Corresponding author: Hyun-Sung Lim

Professor, Smart Electricity, Korea Polytechnic, Gimje, Jeollabuk-do, 54352, Korea. lhs0218@kopo.ac.kr 
Database used consisted of a table containing the measurement values (TMEASUREMENTITEM) and measurement time table(TMEASYREMENTROW). TMEASUREMENTITEM table consisted as bellows.

Table1 TMEASUREMENTITEM table data

\begin{tabular}{cccc}
\hline Table Name & Attribute & Reference data & Key \\
\hline & MEASROWID & bignit & Primary \\
& LOGCHACODE & Char(12) & Primary \\
TMEASUREMENTITEM & MINVAL & Real & - \\
& MAXVAL & Real & - \\
& AVGVAL & Real & - \\
& RMSVAL & real & - \\
\hline
\end{tabular}

TMEASUREMENTROW table consisted as bellows.

Table2 TMEASUREMENTROW table data

\begin{tabular}{cccc}
\hline Table Name & Attribute & Reference data & Key \\
\hline MEASROWID & bignit & Primary \\
MEASUREMENTID & bignit & - \\
TMEASUREMENTROW & MODINFID & Bignit & - \\
& MTIME & datetime & - \\
\hline
\end{tabular}

The entire data was stored in classified data frame and import pandas module from jupyter. Combine the entire data frame into one data frame using the concat function.

\section{Prosumer data classification criteria}

In this paper, the safety standard of prosumer facilities were defined through the technical standard and regulations for electrical equipment. The classification criteria for each prosumer facility are as follows.

Table3 Classification criteria (Photovoltaic system)

\begin{tabular}{ccc}
\hline Item & Description & criteria \\
\hline Frequency & $< \pm 0.2 \mathrm{hz}$ & $59.8<$ Minval, Maxval $<60.2$ \\
Voltage(R Phase) & $< \pm 10 \%$ & $198<$ Minval, Maxval $<242$ \\
Voltage(S phase) & $< \pm 10 \%$ & $198<$ Minval, Maxval $<242$ \\
Voltage(T phase) & $< \pm 10 \%$ & $198<$ Minval, Maxval $<242$ \\
V_THD(R phase) & $<5 \%$ & Minval or Maxval $<5$ \\
V_THD(S phase) & $<5 \%$ & Minval or Maxval $<5$ \\
V_THD(T phase) & $<5 \%$ & Minval or Maxval $<5$ \\
I_THD(R phase) & $<10 \%$ & Minval or Maxval $<10$ \\
I_THD(S phase) & $<10 \%$ & Minval or Maxval $<10$ \\
I_THD(T phase) & $<10 \%$ & Minval or Maxval $<10$ \\
Flicker_s(R & $<1$ & Minval or Maxval $<1$ \\
phase) & $<1$ & Minval or Maxval $<1$ \\
Flicker_s(S & $<1$ & Minval or Maxval $<1$ \\
phase) & $<1$ & Minval or Maxval $<1$ \\
Flicker_s(T & $<1$ & Minval or Maxval $<1$ \\
phase) & $<1$ & Minval or Maxval $<1$ \\
Flicker_l(R & &
\end{tabular}


Table4 Classification criteria (Energy Storage system)

\begin{tabular}{ccc}
\hline Item & Description & criteria \\
\hline Frequency & $< \pm 0.2 \mathrm{hz}$ & $59.8<$ Minval, Maxval $<60.2$ \\
Voltage(R Phase) & $< \pm 10 \%$ & $198<$ Minval, Maxval $<242$ \\
Voltage(S phase) & $< \pm 10 \%$ & $198<$ Minval, Maxval $<242$ \\
Voltage(T phase) & $< \pm 10 \%$ & $198<$ Minval, Maxval $<242$ \\
V_THD(R phase) & $<5 \%$ & Minval or Maxval $<5$ \\
V_THD(S phase) & $<5 \%$ & Minval or Maxval $<5$ \\
V_THD(T phase) & $<5 \%$ & Minval or Maxval $<5$ \\
I_THD(R phase) & $<10 \%$ & Minval or Maxval $<10$ \\
I_THD(S phase) & $<10 \%$ & Minval or Maxval $<10$ \\
I_THD(T phase) & $<10 \%$ & Minval or Maxval $<10$ \\
Flicker_s(R phase) & $<1$ & Minval or Maxval $<1$ \\
Flicker_s(S phase) & $<1$ & Minval or Maxval $<1$ \\
Flicker_s(T phase) & $<1$ & Minval or Maxval $<1$ \\
Flicker_l(R phase) & $<1$ & Minval or Maxval $<1$ \\
Flicker_l(S phase) & $<1$ & Minval or Maxval $<1$ \\
Flicker_1(T phase) & $<1$ & Minval or Maxval $<1$ \\
Temperature & $-20^{\circ} \mathrm{C}<$ temp $<40^{\circ} \mathrm{C}$ & $-20>$ Minval or Maxval $<40$ \\
Humidity & $<80 \%$ & Maxval $<80$ \\
\hline
\end{tabular}

Table5 Classification criteria (EV charger system)

\begin{tabular}{|c|c|c|}
\hline Item & Description & criteria \\
\hline Frequency & $< \pm 0.2 \mathrm{hz}$ & $59.8<$ Minval, Maxval $<60.2$ \\
\hline Voltage(R Phase) & $< \pm 10 \%$ & $198<$ Minval, Maxval $<242$ \\
\hline Voltage(S phase) & $< \pm 10 \%$ & $198<$ Minval, Maxval $<242$ \\
\hline Voltage(T phase) & $< \pm 10 \%$ & $198<$ Minval, Maxval $<242$ \\
\hline V_THD(R phase) & $<5 \%$ & Minval or Maxval $<5$ \\
\hline V_THD(S phase) & $<5 \%$ & Minval or Maxval <5 \\
\hline V_THD(T phase) & $<5 \%$ & Minval or Maxval $<5$ \\
\hline I_THD(R phase) & $<10 \%$ & Minval or Maxval $<10$ \\
\hline I_THD(S phase) & $<10 \%$ & Minval or Maxval $<10$ \\
\hline I_THD(T phase) & $<10 \%$ & Minval or Maxval $<10$ \\
\hline Flicker_s(R phase) & $<1$ & Minval or Maxval <1 \\
\hline Flicker_s(S phase) & $<1$ & Minval or Maxval <1 \\
\hline Flicker_s(T phase) & $<1$ & Minval or Maxval <1 \\
\hline Flicker_1(R phase) & $<1$ & Minval or Maxval <1 \\
\hline Flicker_l(S phase) & $<1$ & Minval or Maxval <1 \\
\hline Flicker_1(T phase) & $<1$ & Minval or Maxval <1 \\
\hline
\end{tabular}


Table6 Classification criteria (Wind power system)

\begin{tabular}{|c|c|c|}
\hline Item & Description & criteria \\
\hline Frequency & $< \pm 0.2 \mathrm{hz}$ & $59.8<$ Minval, Maxval $<60.2$ \\
\hline Voltage(R Phase) & $< \pm 10 \%$ & $198<$ Minval, Maxval $<242$ \\
\hline Voltage(S phase) & $< \pm 10 \%$ & $198<$ Minval, Maxval $<242$ \\
\hline Voltage(T phase) & $< \pm 10 \%$ & $198<$ Minval, Maxval $<242$ \\
\hline V_THD(R phase $)$ & $<5 \%$ & Minval or Maxval $<5$ \\
\hline V_THD(S phase) & $<5 \%$ & Minval or Maxval <5 \\
\hline V_THD(T phase) & $<5 \%$ & Minval or Maxval $<5$ \\
\hline I_THD(R phase) & $<10 \%$ & Minval or Maxval $<10$ \\
\hline I_THD(S phase) & $<10 \%$ & Minval or Maxval $<10$ \\
\hline I_THD(T phase) & $<10 \%$ & Minval or Maxval $<10$ \\
\hline Flicker_s(R phase $)$ & $<1$ & Minval or Maxval <1 \\
\hline Flicker_s(S phase) & $<1$ & Minval or Maxval <1 \\
\hline Flicker_s(T phase) & $<1$ & Minval or Maxval <1 \\
\hline Flicker_1(R phase) & $<1$ & Minval or Maxval <1 \\
\hline Flicker_l(S phase $)$ & $<1$ & Minval or Maxval <1 \\
\hline Flicker_1(T phase) & $<1$ & Minval or Maxval <1 \\
\hline Wind Speed & $<25 \mathrm{~m} / \mathrm{s}$ & Maxval $<25$ \\
\hline
\end{tabular}

For data classification, a column called label was created in the integrated data frame. After column generation, each data labeling was carried out. By comparing the measurements of MEASROWID and each LOGCGACODE, the data entered the normal results within the normal classification criteria $($ label $=1)$. Abnormal results were entered as label 0. After comparing the labels of each LOGCHACODE, if the whole label is normal, enter label is 1 or abnormal data enter label is 0. [Figure 2] shows an example of the results determined by labelling the data.

\begin{tabular}{|c|c|c|c|c|c|c|c|c|c|c|c|c|}
\hline & MEASROWID_O & LOGCHACODE_O & MINVAL_O & MAXVAL_0 & label_o & & MINVAL_1 & MAXVAL_1 & label_1 & LOGC & ... label_16 & \\
\hline 0 & 1 & $01010 E 800100$ & 0.00000 & 0.00000 & 1 & 10120810000 & 0.0000 & 0.0000 & 1 & 10134810000 & - & 1 \\
\hline 1 & 2 & 01010E800100 & 0.00000 & 0.00000 & 1 & 10120810000 & 0.0000 & 0.0000 & 1 & 10134810000 & - & 1 \\
\hline 2 & 3 & $01010 E 800100$ & 60.00005 & 60.00009 & 1 & 10120810000 & 220.2858 & 220.2859 & 1 & 10134810000 & $\ldots$ & 1 \\
\hline 3 & 4 & $01010 E 800100$ & 60.00005 & 60.00009 & 1 & 10120810000 & 220.2858 & 220.2859 & 1 & 10134810000 & $\ldots$ & 1 \\
\hline 4 & 5 & O1010E800100 & 60.00005 & 60.00009 & 1 & 10120810000 & 220.2858 & 220.2859 & 1 & 10134810000 & - & 1 \\
\hline 5 & 6 & $01010 E 800100$ & 60.00005 & 60.00009 & 1 & 10120810000 & 220.2858 & 220.2860 & 1 & 10134810000 & - & 1 \\
\hline 6 & 7 & O1010E800100 & 60.00005 & 60.00009 & 1 & 10120810000 & 220.2858 & 220.2859 & 1 & 10134810000 & - & 1 \\
\hline 7 & 8 & 01010E800100 & 60.00005 & 60.00009 & 1 & 10120810000 & 220.2858 & 2202859 & 1 & 10134810000 & $\ldots$ & 1 \\
\hline 8 & 9 & $01010 E 800100$ & 60.00005 & 60.00009 & 1 & 10120810000 & 220.2858 & 220.2859 & 1 & 10134810000 & -. & 1 \\
\hline 9 & 10 & $01010 E 800100$ & 60.00005 & 60.00009 & 1 & 10120810000 & 220.2858 & 220.2859 & 1 & 10134810000 & - & 1 \\
\hline 10 & 11 & $01010 E 800100$ & 60.00005 & 60.00009 & 1 & 10120810000 & 220.2858 & 220.2859 & 1 & 10134810000 & - & 1 \\
\hline 11 & 12 & $01010 E 800100$ & 60.00005 & 60.00009 & 1 & 10120810000 & 220.2858 & 220.2859 & 1 & 10134810000 & 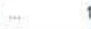 & 1 \\
\hline 12 & 13 & $01010 E 800100$ & 60.00005 & 60.00009 & 1 & 10120810000 & 220.2858 & 220.2859 & 1 & 10134810000 & $\ldots$ & 1 \\
\hline 13 & 14 & O1010E800100 & 60.00005 & 60.00009 & 1 & 10120810000 & 220.2858 & 220.2859 & 1 & 10134810000 & ... & 1 \\
\hline 14 & 15 & $01010 E 800100$ & 60.00005 & 60.00009 & 1 & 10120810000 & 220.2858 & 220.2860 & 1 & 10134810000 & ... & 1 \\
\hline
\end{tabular}

Figure 2 Labelling data result example 


\section{Results and Discussion}

This paper applied labeled data to AI algorithms, verification through DNN(Deep Neural Network) method. DNN is an artificial neural network consisting of several layers of concealment between the input and output layers. In general, complex nonlinear relationships can be modeled. DNN is useful in areas such as classification numerical prediction, image training and literacy.

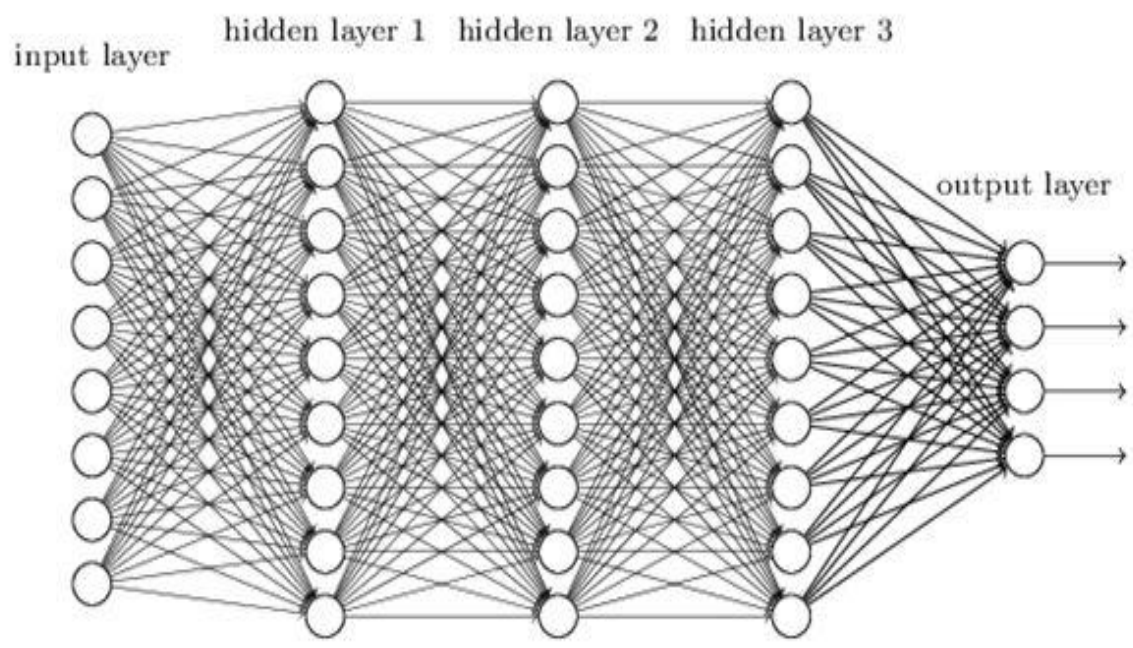

Figure 3 Standard structure of DNN

Methods for applying DNN are as follows.

- Divide entire data in training sets and test sets

- Normalized deviation between data is between 0 and 1 using MinMaxScaler

- Creating deep learning model

- Extract predictive results to the created model

For analyzing the accuracy of the generated model, this paper used Precision, Recall, Accuracy, F1-score. Precision is the fraction of relevant instances among the retrieved instances. Recall is the fraction of the total amount of relevant instances that were actually retrieved [9 10]. Accuracy is closeness of the measurements to a specific value. The f1-score is the harmonic mean of Precision and Recall has a value from 0 to 1 . Each expression is expressed as follows.

$$
\begin{aligned}
& \text { Precision }=\frac{T P}{T P+F P} \\
& \text { Recall }=\frac{T P}{T P+F N} \\
& \text { Accuracy }=\frac{T P+T N}{T P+F N+F P+T N} \\
& \mathrm{~F} 1-\text { score }=2 \times \frac{\text { Precision } \times \text { Recall }}{\text { Precision }+ \text { Recall }}
\end{aligned}
$$

Experiment data used from Energy storage system. DNN analysis results shows as Table 7.

Table7 Result of DNN analysis

\begin{tabular}{ccccc}
\hline Class & Precision & Recall & F1-score & Support \\
\hline 0 & 0.92 & 0.98 & 0.95 & 310 \\
1 & 1.00 & 0.99 & 0.99 & 1,755 \\
Accuracy & & & 0.98 & 2.065 \\
Macro avg & 0.96 & 0.98 & 0.97 & 2.065 \\
Weighted avg & 0.99 & 0.98 & 0.99 & 2.065 \\
\hline
\end{tabular}




\section{Conclusion}

This paper presented database structure of prosumer systems (Photovoltaic system, ESS system, Wind Power system, EV charger system). The safety standard of prosumer facilities were defined through the technical standard and regulation for electrical equipment. The classification criteria for each prosumer facility. By comparing the measurement data and each logical code, the data entered the normal results is label 0 or abnormal data results is label 0 . And then all criteria data compared each other, whole label data is normal entered label 1 or abnormal entered label 0. For applied AI algorithms, this paper adapted DNN(Deep Neural Network) method. This paper used Precision, Recall, Accuracy, F1-score. The result of experiment is high value of each expression. Especially, Recall value is high values and this expression is important to safety area focus. This database structure will provide online prosumer platform system and better prediction safety system.

\section{Acknowledgment}

This work was supplied by the Korea Institute for Advancement of Technology and the Ministry of Trade Industry \& Energy (MOTIE) of Republic of Korea (NO. 20172210200030)

\section{References}

1. A.J.D Rathnayaka. Analysis of energy behavior profiles of prosumers. IEEE 10th International Conference on Industrial informatics. 2012 July

2. Keyhani, Design of Smart Power Grid Renewable Energy stems, Wiley, 2011.

A. J. D. Rathnayaka, V. M. Potdar, O. Hussain and T. Dillon, "identifying prosumer's energy sharing behaviours for forming optimal prosumer-communities", Int. Conf. Cloud Serv. Comput., 2011.

3. P. G. D. Silva, S. Karnouskos and D. Ilic. A Survey Towards understanding Residential Prosumers in Smart Grid Neighborhoods in innovative Smart Grid Technologies Europe. ISGT Europe. 2012.

4. Asif Raza Butt. Energy sharing and management for prosumers in smart grid with integration of storage system. ICSG. 2017

5. CamposInês, Pontes Luz Guilherme, Marín-González Esther, Gährs Swantje, Hall Stephen, Holstenkamp Lars. Regulatory challenges and opportunities for collective renewable energy prosumers in the EU. Energy policy. 2020;138

6. T. Bauwens, P. Devine-Wright. Positive energies : An empirical study of community energy

7. participation and attitudes to renewable energy. Energy Policy. 2018;118;612-625

8. D. Brown, S. Hall, M.E. Davis. Prosumers in the post subsidy era: an exploration of new

9. prosumer business models in the UK. Energy Policy. 2019;135;110984

10. Y. LeCun, Y. Bengio, and G. Hinton, Deep learning. Nature.2015;521;7553;436-444

11. D. Silver, A. Huang, C. J. Maddison et al. Mastering the game of Go with deep neural

12. networks and tree search. Nature.2016;529;7587;484-489

13. Satapathy, S. K., Mishra, S., Mallick, P. K., Badiginchala, L., Gudur, R. R., \& Guttha, S. C. (2019). Classification of Features for detecting Phishing Web Sites based on Machine Learning Techniques. International Journal of Innovative Technology and Exploring Engineering, volume8 (8S2), 425-430.

14. Monika, P., Rao, P. V., Premkumar, B. C., \& Mallick, P. K. (2020). Implementing and Evaluating the Performance Metrics Using Energy Consumption Protocols in MANETs Using Multipath RoutingFitness Function. In Cognitive Informatics and Soft Computing (pp. 281-294). Springer, Singapore.

15. Satapathy, S. K., Mishra, S., Sundeep, R. S., Teja, U. S. R., Mallick, P. K., Shruti, M., \& Shravya, K. (2019). Deep learning based image recognition for vehicle number information. International Journal of Innovative Technology and Exploring Engineering, 8, 52-55. 